\title{
Performance analysis of 802.11ac with frame aggregation using NS3
}

\author{
N. Khalil, A. Najid \\ Telecommunication Systems, Networks and Services Laboratory, \\ National Institute of Posts and Telecommunications (INPT), Morocco
}

\section{Article Info \\ Article history: \\ Received Dec 25, 2018 \\ Revised Apr 25, 2020 \\ Accepted May 8, 2020 \\ Keywords: \\ Channel bonding \\ Frame aggregation \\ IEEE 802.11ac \\ WLAN}

\begin{abstract}
$802.11 \mathrm{ac}$ is an interesting standard of IEEE bringing multiple advantages than its predecessor $802.11 \mathrm{n}$. 802.11ac is faster and more scalable version of 802.11n offering the capabilities of wireless Gigabit Ethernet. 802.11ac will enable access points (AP) to support more STAs with a better experience for clients and more channel bonding increasing from a maximum of $40 \mathrm{MHz}$ with $802.11 \mathrm{n}$ up to 80 or $160 \mathrm{MHz}$ with 802.11 ac standard. In this paper, we analyze and evaluate the $802.11 \mathrm{ac}$ performance using NS3 simulator (v3.26) relying on several features like channel bonding, modulation and coding schemes, guard interval and frame aggregation. Then, we presentthe effect of the variation of $d$ istance between STAs and AP on the network performance in term of throughput. Finally, we capture the most relevant simulations outcomes and we indicate some research challenges for the future work.
\end{abstract}

Copyright $(0) 2020$ Institute of Advanced Engineering and Science. All rights reserved.

\section{Corresponding Author:}

Nabil Khalil,

Telecommunication Systems, Networks and Services Laboratory,

National Institute of Posts and Telecommunications (INPT),

Avenue Allal Al Fassi, Rabat, Morocco.

Email: nabil.khali102@gmail.com

\section{INTRODUCTION}

The IEEE 802.11ac is a Very High Throughput standard operating only in $5 \mathrm{MHz}$ frequency band. By using $5 \mathrm{GHz}$ frequency band, 802.11ac avoids consequently much of the interference due to the important use of $2.4 \mathrm{GHz}$ ISM band in the three ITU regions. The new features and enhancements in the MAC and PHY layers of 802.11ac allowed this technology to achieve data rates approaching 7Gbps. 802.11ac outperform the $802.11 \mathrm{n}$ data rates by introducing several improvements like wider channels 80 and 160 $\mathrm{MHz}$, also 256QAM modulation enhanced the number transmitted bits from six bits (64QAM) to eight bits on each subcarrier in the channel and raised the maximum number of spatial streams (SS) on the access point side to eight SSs instead of four in $802.11 \mathrm{n}$ while being able to transmit to multiple clients at the same time as a result of MU-MIMO feature.

The study of IEEE 802.11 standard performance is the main subject of multiple researches by means of analytical models or simulation. An important analysis was first introduced in [1] as Bianchi presented the performance of wireless networks using 802.11 distributed coordination function (DCF) based on a Markovian model. Also, in [2], an analytical model was presented in order to analyze the impact of basic and RTS/CTS access mechanisms of DCF on 802.11ac throughput. The results based on the throughput equation in [2] have showed interesting outcomes about the effectiveness of RTS/CTS mechanisms when packet size increases and the importance of frame aggregation which was also studied in [2, 3]. In [4], the author presents only BER values for different modulation techniques BPSK, QPSK, 16QAM and 256QAM revealing that it is preferable using the BPSK modulation rather than 256QAM with higher BER in transmitting the data on the AWGN channel. Moreover, in [5], an overview of the main performance of both 802.11 ac and 802.11n was presented where the authors noticed that, in terms of performance, the two 802.11 
protocols aren't much different in case of 20 or $40 \mathrm{MHz}$ channel width. Then, the study of frame aggregation feature effects was projected in their future work. In [6], the author proposes a method to give an estimation of average delay of MPDUs for each queue by using A-MPDU aggregation and allow to determine an optimal number of aggregated MPDUs in the queue in order to maximize the system throughput. Furthermore, in [7] the author defines wireless local area networks (WLAN) performance in terms of throughput as the amount of information that is transmitted from the emitter to the receptor per time unit without losses in the packets sent. In this paper, we analyze the performance of 802.11ac based on a realistic scenario where three networks are simulated with different frame aggregation schemes (A-MSDU, A-MPDU, A-MPDU of A-MSDU) and channel bandwidths. The rest of the paper is organized as follows: In section 2, we present the main enhancements of 802.11ac standard, then in section 3 we give an overview of the NS3 simulator along with the network model. Furthermore, in section 4, we present the achieved results and we analyze the performance of 802.11ac with different channel bandwidths, MCSs and frame aggregation schemes. Finally, section 5 concludes the paper.

\section{IEEE 802.11ac TECHNOLOGY BACKGROUND}

In this section we review the amendment of IEEE 802.11ac together with the PHY and MAC layer enhancements. This survey focusses on the main techniques and aspects making the IEEE 802.11ac performance more interesting.

\subsection{IEEE 802.11ac PHY main features \\ 2.1.1. Channel bandwidth}

IEEE 802.11ac [8] is a 5GHz-only technology allowing STAs to operate in a less loaded wireless network, however dual-band APs and STAs can always use their $802.11 \mathrm{n}$ interface to operate in the $2.4 \mathrm{GHz}$ ISM frequency band. 802.11ac standard introduces channel bonding by increasing the maximum $802.11 \mathrm{n}$ bandwidth size $40 \mathrm{MHz}$ to $80 \mathrm{MHz}$ or even $160 \mathrm{MHz}$. In case of a $160 \mathrm{MHz}$ channel, contiguous spectrum becomes hard to achieve, the standard introduces two forms of $160 \mathrm{MHz}$ channels ; a one continuous block and an " $80+80$ " channel by combining two 80 channel widths [8]. An increased value of $117 \%$ in data rates is noticed by moving from $40 \mathrm{MHz}$ to $80 \mathrm{MHz}$ and $333 \%$ with a $160 \mathrm{MHz}$ channel width [9].

\subsubsection{Modulation and coding scheme}

Compared to its predecessor $802.11 \mathrm{n}, 802.11 \mathrm{ac}$ makes evolutionary improvements to modulation and coding scheme (MCS). The highest 802.11n modulation and coding scheme is 64QAM with a 5/6 coding rate which has enhanced in IEEE 802.11ac up to 256-QAM and a 5/6 coding rate allowing an eight information bits in each symbol instead of six in the 802.11n (a combination of data and error correction bits). However, the 256-QAM in 802.11ac requires a bigger signal-to-noise ratio on the receiver part to preserve a low bit-error probability compared to other modulation schemes [10]. As we can see in the below Table 1, the choice of the MCS is simpler in 802.11ac by offering only 10 options rather than the 70-plus options offered by $802.11 \mathrm{n}[9]$ :

Table 1. MCS values for 802.11ac

\begin{tabular}{ccc}
\hline MCS index value & Modulation & Code rate \\
\hline 0 & BPSK & $1 / 2$ \\
1 & QPSK & $1 / 2$ \\
2 & QPSK & $3 / 4$ \\
3 & 16-QAM & $1 / 2$ \\
4 & 16-QAM & $3 / 4$ \\
5 & 64-QAM & $2 / 3$ \\
6 & 64-QAM & $3 / 4$ \\
7 & 64-QAM & $5 / 6$ \\
8 & 256-QAM & $3 / 4$ \\
9 & 256-QAM & $5 / 6$ \\
\hline
\end{tabular}

The support of the first seven modulation and coding schemes is mandatory, however the 256-QAM modulation supported by the majority of the 802.11ac products in the market can offer more data within the transmission. Also, the 802.11ac standard makes the selection of the MCS more simpler due to the fact that this parameter is no longer tied to the number of spatial streams as it is with its predecessor 802.11n. The MCS index value along with the number of streams to produce an overall data rate defines the link speed. 


\subsubsection{Beamforming and multi-user MIMO (MU-MIMO)}

One of the major enhancements of 802.11ac standard is the Multi-user MIMO and it is among the most crucial techniques that lead WLANs towards the gigabit era. The MU-MIMO technology has been embraced as an essential element by the latest wireless standards [11]. Prior to 802.11ac, each transmission was performed to a single destination only because of the 802.11 standards were in a single-user mode. Compared to its predecessors, 802.11ac MU-MIMO holds the following advantages: (1) Increased Throughput. In case of a SU-MIMO, the theoretical capacity gain can be expressed by a multiplicative factor of $\min \{\mathrm{Ntr} ; \mathrm{Nrc}\}$ where $\mathrm{Ntr}$ and $\mathrm{Nrc}$ are the number of transmitting and receiving antennas [12, 13], although with MU-MIMO, this multiplicative factor is extended to $\min \{\mathrm{aNtr}$; bNrc $\}$ where $\mathrm{a}$ and $\mathrm{b}$ represent the number of simultaneous transmitters and receivers; (2) Increased diversity gain. STAs spatially distributed make MU-MIMO system more resistant to channel rank loss and antenna correlation [14] (3) reduced terminal cost. Spatially distributed STAs even equipped only with a single antenna are supported by the MU-MIMO system in order to exchange information at the same time with the access point, allowing better development of low-cost user terminals [15]. However, in [16] the author shows also that MU-MIMO may be less performant as SISO when using channel calibration for every transmission considering that the performance of MU-MIMO depends on calibration procedure overhead. Also, Beamforming is a technique used occasionally in networks to improve the signal to noise ratio (SNR) at the receiver part, allowing increased values of data rates. In case of two receivers placed in different positions, a beamformed transmission could be sent to each of them at the same time [8].

\subsubsection{Spatial multiplexing}

802.11ac standard extended the maximum number of spatial streams to eight instead of four streams with 802.11n (Single user-MIMO) [17] providing transmissions with higher data rates at the same time for several clients. Also, as seen in [18], by using different spatial streams 1x1, 2x2, 3x3 and 4x4 configuration, just with $802.11 \mathrm{n}$ standard, the average gradually increases as the network is loaded with data.Two rules were defined to limit the MU-MIMO transmission to become more controllable: The number of simultaneous beams towards different clients is up to four implying a maximum number of simultaneous receivers of MU-MIMO limited to four and a maximum number of spatial streams inside a beam directed to a client is also four [10].

The throughput is directly proportional to the number of spatial stream. As the number of spatial stream increases more antennas and RF chains in transmitter and receiver part will be required which implies additional power consumption. Due to this factor, some devices could limit their number of antennas to a maximum of three [19]. Furthermore, as seen in the above through its main features, the standard IEEE 802.11ac can offer exceptional experience to different clients from an IEEE 802.11ac access point compared to its predecessor. Figure 1 summarizes the main difference between IEEE802.11ac and 802.11n [19].

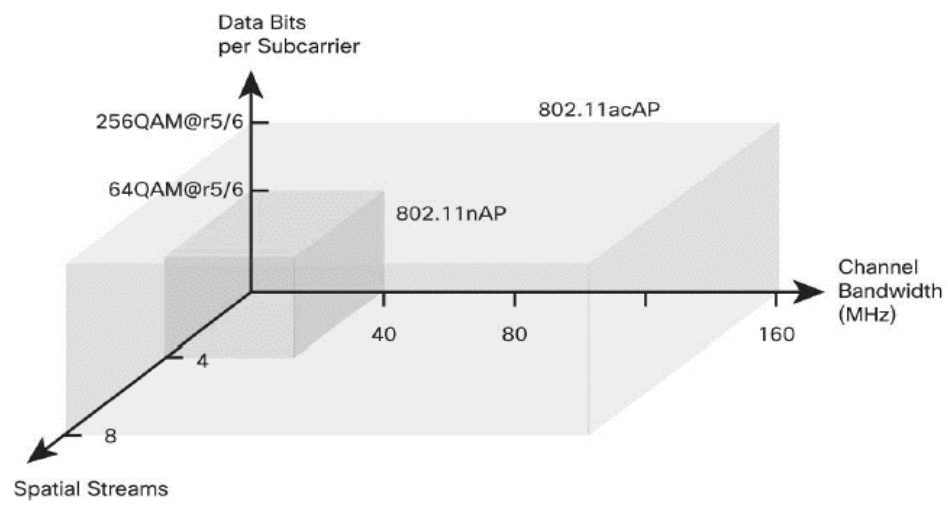

Figure 1. The 802.11ac and 802.11n main features

\subsubsection{Guard interval}

The time between transmitted symbols is Guard Interval. For a good performance, the symbol should arrive at the receiver part without interference or noise, thus assuring proper decoding and fewer errors. When the delay between different RF paths toward the receiver exceeds the guard interval an inter-symbol interference occurs [5]. Guard interval has the same effect with 802.11ac as in 802.11n standard. 802.11ac can select a shortened OFDM guard interval by moving it from 800ns to 400ns if both the transmitter and the receiver are capable of processing it which provides a $10 \%$ boost in throughput [8]. 


\subsection{IEEE 802.11ac MAC main features}

\subsubsection{Frame aggregation}

IEEE 802.11 ac conserve many of the enhancements that were introduced in IEEE 802.11n like the frame aggregation feature. The major idea behind frame aggregation concept is that we can concatenate a number of frames together so that overheads can be added over a group of frames rather than over distinct ones. Since 2009, when the 802.11n standard was introduced, WLANs use aggregation to enhance their efficiency [20]. Additionally, a backoff procedure will not be needed for each single frame. A-MSDU contains MSDUs with a common MAC header then it is encapsulated in MPDU and A-MPDU contains MPDUs with different MAC headers. 802.11ac, however, adds an interesting new take on aggregation, all frames transmitted use the aggregate MPDU (A-MPDU) format and all 802.11ac data frames will be sent in an A-MPDU aggregation scheme, even if the A-MPDU has an only one frame [8]. The mechanism avoids overheads like multiple DCF interframe spacing (DIFS), short interframe spacing (SIFS), and backoff intervals, thus saves channel time and allows higher throughput [21]. The size of A-MSDU and A-MPDU defined by the IEEE $802.11 \mathrm{ac}$ is respectively 11406 bytes and 1048575 bytes. Frame aggregation is very helpful in enhancing channel utilization further and increasing efficiency of a wireless station [22]. Figure 2 presents the A-MPDU frame format:

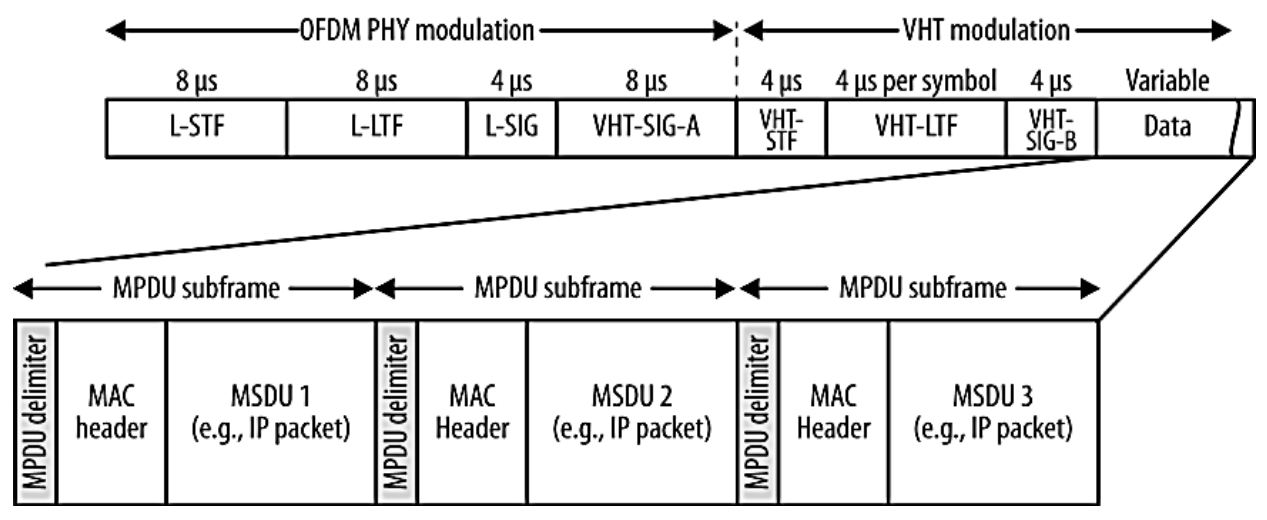

Figure 2. A-MPDU frame format

\subsubsection{Block acknowledgment}

Sending an acknowledgement (ACK) [5] from a receiving station in the predecessor standard 802.11 $\mathrm{a} / \mathrm{b} / \mathrm{g}$ is done almost instantaneously to approve the reception of the frames. This mechanism allows enhancing the performance and strength of 802.11 networks by ensuring that all sent frames eventually get to the receiver device. However, transmitting multiple frames may reduce the efficiency of the protocol due to added ACK frames for each transmission. The block acknowledgement (BA) mechanism brings an enhancement to this issue by sending a single block ACK frame after several received frames which enhances the performance of the transmission. The BA was first introduced in 802.11e as an optional scheme to improve the MAC efficiency. The BA mechanism was enhanced by $802.11 \mathrm{n}$ amendment and then made obligatory for the different $802.11 \mathrm{n}$ capable devices. Several MPDUs can then be acknowledged together by a single block acknowledgement frame rather than transmitting separate ACK for each MPDU. Block ACK also exploits the benefits of MPDU aggregation by acknowledging several frames within a single transmission opportunity (TxOP) period [23].

\section{NETWORK MODEL AND SIMULATOR}

In the present paper, the ns3-3.26 version is used to conduct the performance analysis of $802.11 \mathrm{ac}$ using different schemes of frame aggregation (A-MPDU, A-MSDU and hybrid aggregation A-MPDU of A-MSDU). This version of ns3 was first released in October 2016 with a major enhancement being introduced to the Wifi module. More details about the ns-3 simulator and its capabilities could be found in $[24,25]$.

In order to evaluate the performance of different schemes of frame aggregation, A-MPDU, A-MSDU and A-MPDU of A-MSDU (hybrid aggregation) in terms of channel throughput based on different values of MCS and channel width $(20,40,80$ or $160 \mathrm{MHz})$, we built the ns3 model based on the Wi-Fi Module. Three 802.11ac infrastructures were built where each one supports a one STA that is connected to 
an Access point (BSS infrastructure). Multiple scenarios were tested based on the type of aggregation affected to each BSS. Furthermore, the evaluation of the UDP throughput was also performed in terms of distance between the STA and the access point (AP) in order to define the most appropriate MCS for the different ranges. The parameters considered for the performance evaluation of the ns 3 model are listed below in Table 2:

Table 2. Simulation parameters

\begin{tabular}{ll}
\hline Parameter & Description \\
\hline Standard & $802.11 \mathrm{ac}$ \\
Aggregation scheme & A-MPDU, A-MSDU, A-MPDU of A-MSDU \\
& (Hybrid) \\
Mobility model & Constant position mobility model \\
Payload size & 1472 bytes \\
Guard interval & Short GI, Long GI \\
Channel width & $20,40,80,160 \mathrm{MHz}$ \\
\hline
\end{tabular}

\section{RESULTS AND DISCUSSION}

In this paper, we first analyze the IEEE 802.11ac throughput for different values of the modulation and coding scheme taking in consideration the variation of the channel bandwidth values (20, 40, 80 and $160 \mathrm{MHz}$ ). This first evaluation will be performed for both short and long guard interval respectively shown in Figures 3 and 4. Then we analyze the network performance under various MCS values with different channel bandwidths 40,80 and $160 \mathrm{MHz}$ in Figure 5, 6 and 7. Finally, we evaluate the impact of the distance between STA and the access point on throughput and we show the variation of the network performance based on distance and different MCS values and the tradeoff between these two parameters, Figures 8 and 9. All these simulations are based on the parameters set in Table 2.

\subsection{Throughput based on channel bandwidth and guard interval}

As seen in section 2, shrinking the guard interval from $800 \mathrm{~ns}$ to $400 \mathrm{~ns}$, boost the throughput values, as we can also observe with the ns 3 simulation results as shown in Figures 2 and 3, based on the model described above. The guard interval allows eliminating the inter-symbol interference and by reducing this interval to the half we increase the throughput.

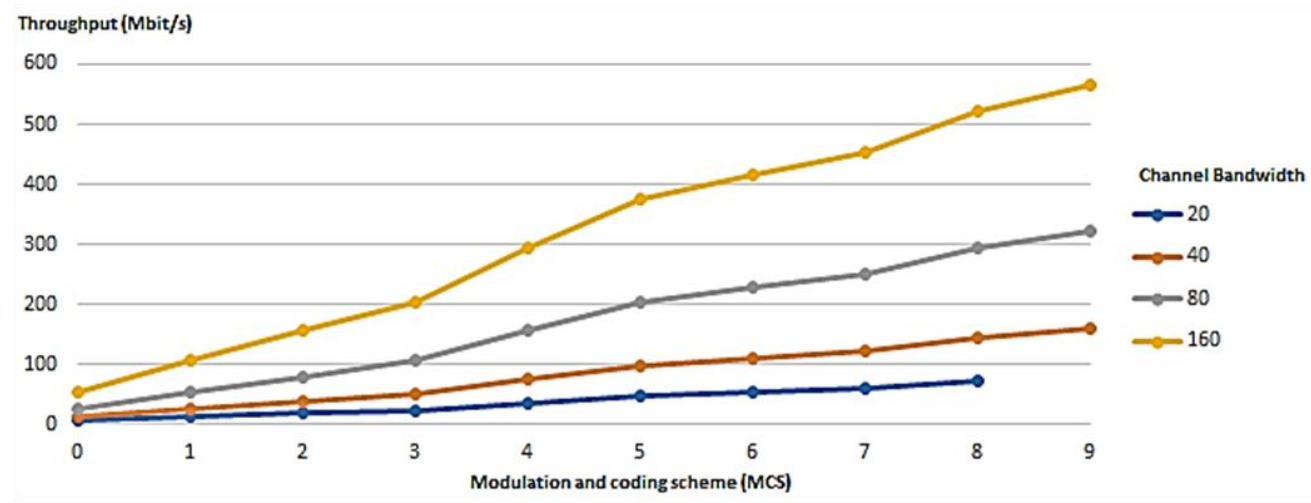

Figure 3. 802.11ac throughput with long guard interval

The results in Figures 3 and 4 show a variation of throughput based on the MCS values for 20, 40, 80 and $160 \mathrm{MHz}$ channel bandwidth. The throughput with channel bonding when bandwidth is $160 \mathrm{MHz}$ is highly exceeding the throughput with $802.11 \mathrm{ac}$ through a $80 \mathrm{MHz}$ channel width and approaching the value of $600 \mathrm{Mbit} / \mathrm{s}$ for 256QAM, 5/6 coding rate (MCS9) and one spatial stream. Also, as the MCS values increases we note that the throughput is becoming more important as a result of higher modulations and codes rates. Higher modulations allow more data in the transmission but they require also higher signal-tonoise ratios. Additionnally, higher code rates have more data and less redundancy with a risk of not overcoming as many errors. Moreover, a combination of the $20 \mathrm{MHz}$ channel bandwidth, MCS 9 and one spatial stream is not allowed according to 802.11ac standard specification like shown in the Figures 3 and 4. 


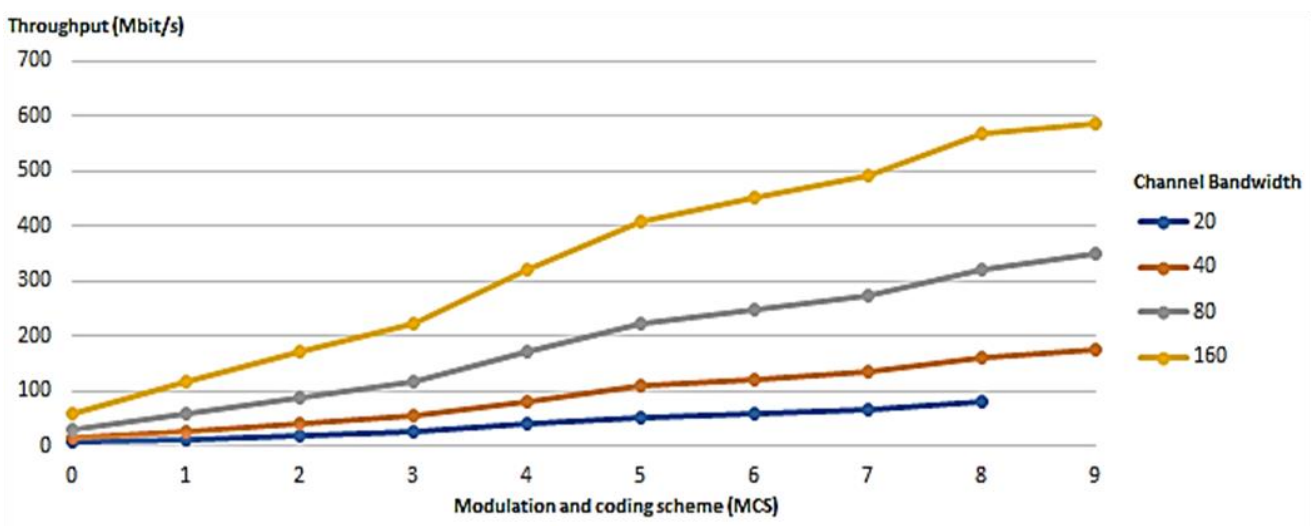

Figure 4. 802.11ac throughput with short guard interval

\subsection{Throughput based on channel bandwidth and frame aggregation}

To investigate the performance of the 802.11ac network with various types of frame aggregation, we create three scenarios where we can explore the impact of A-MPDU, A-MSDU and A-MPDU of A-MSDU on throughput based on three channel bandwidths 40, 80 and $160 \mathrm{MHz}$ and different values of MCS. In Figures 5-7, throughput is linear between MCS 0 and MCS 3 for the selected bandwidths with values of throughputs varying from $13.87 \mathrm{Mbit} / \mathrm{s}(\mathrm{MCS} 0,20 \mathrm{MHz}$ ) to $600 \mathrm{Mbit} / \mathrm{s}$ (MCS7,160Mhz). Also, we can observe that for the chosen A-MPDU of A-MSDU (Hybrid) frame aggregation scheme, the values of throughput are the highest for the three-channel width 40, 80 and $160 \mathrm{MHz}$.

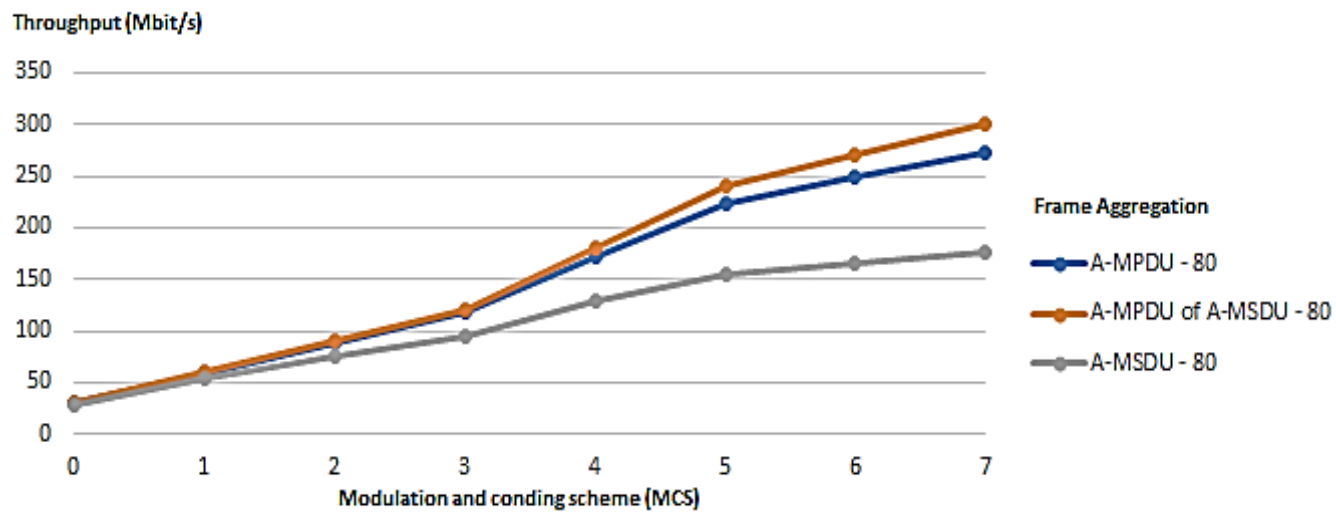

Figure 5. Throughput for $40 \mathrm{MHz}$ channel bandwidth

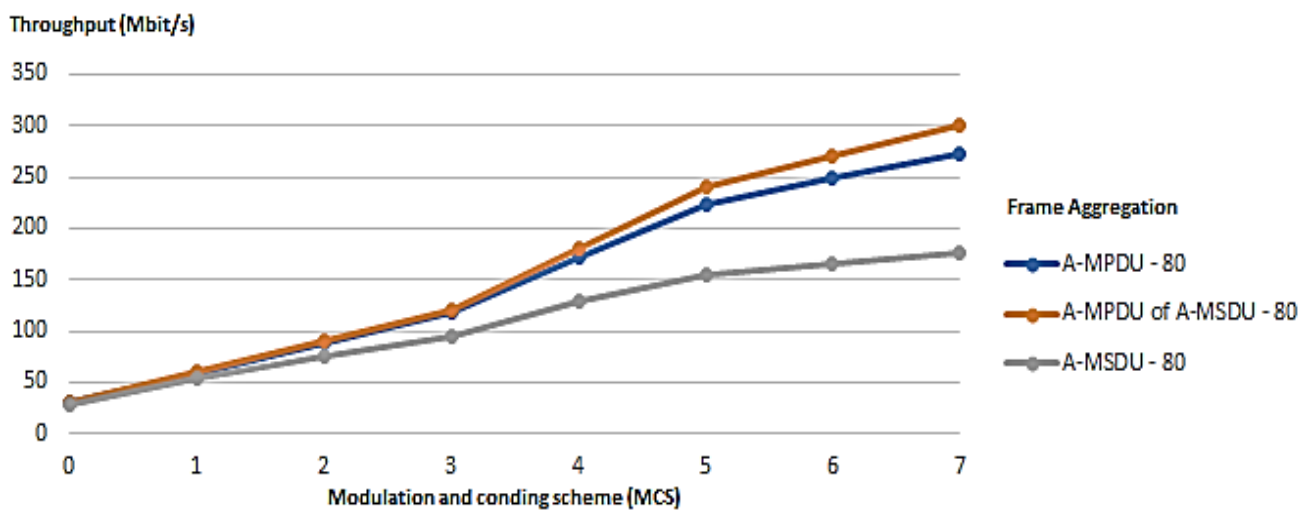

Figure 6. Throughput for $80 \mathrm{MHz}$ channel bandwidth 


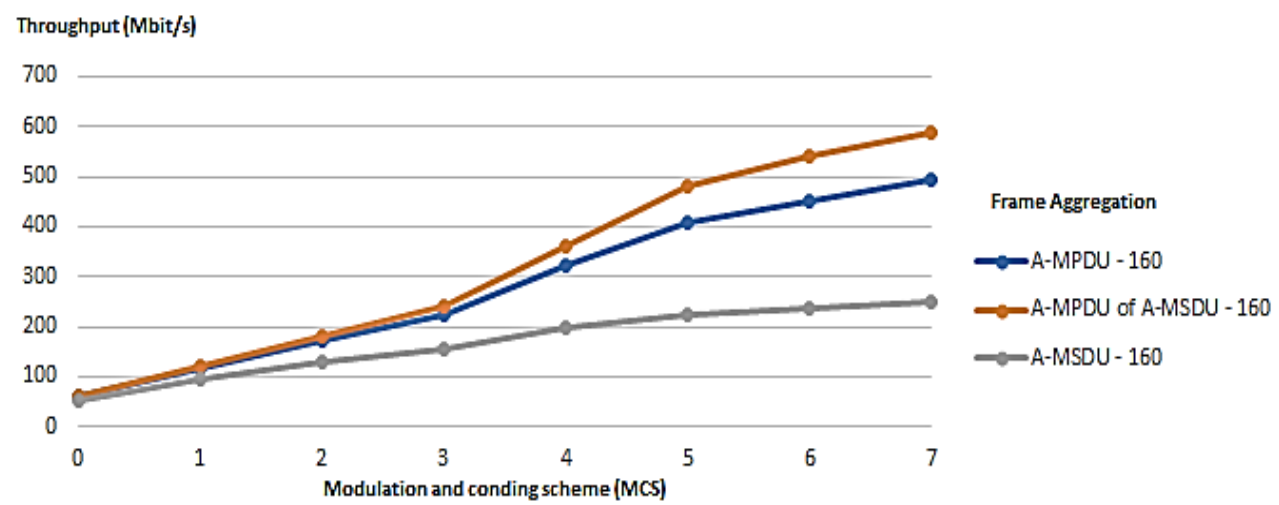

Figure 7. Throughput for $160 \mathrm{MHz}$ channel bandwidth

With A-MSDU frame, multiple MSDUs can be aggregated into a single frame allowing more transmitted data bit per MAC and PHY header. This mechanism is good for efficiency but it makes the retries on the distinct MSDU level impossible. Although, A-MPDU surpasses the A-MSDU aggregation scheme primary due to the large size and the retransmission capacities of a subframe in erroneous channels, it is also limited due to the number of subframe headers compared to hybrid aggregation (A-MPDU of A-MSDU). As seen in Figures 5-7, the hybrid aggregation outperforms all the other frame aggregation schemes performance in terms of throughput mainly due to reduction of number of MPDU subframe headers.

\subsection{Impact of distance on the network performance}

In Figure 8, we plot the variation of throughput with different 802.11ac MCSs and for several ranges $5,10,15,20,25,30$ and $35 \mathrm{~m}$ which allows us to show the impact of the distance between the STA and access point on the performance of the network in term of throughput which decreases tremendously for a specific modulation and coding rates. Thus, we can clearly see that the throughput is massively decreasing when we force a specific MCS and the use of a 256QAM modulation (MCS 8 or 9) is no longer efficient after a range of $10 \mathrm{~m}$. However, we can observe that for better performance in longer ranges like $35 \mathrm{~m}$, QPSK modulation is considered better. Also, in Figure 9, we plotted the variation of throughput for each channel bandwidths for a fixed distance. We observe that the throughput is almost linear till MCS6 and decrease for MCS7 and 160MHz channel bandwidth due to channel conditions and the longer distance effect between STA and AP.

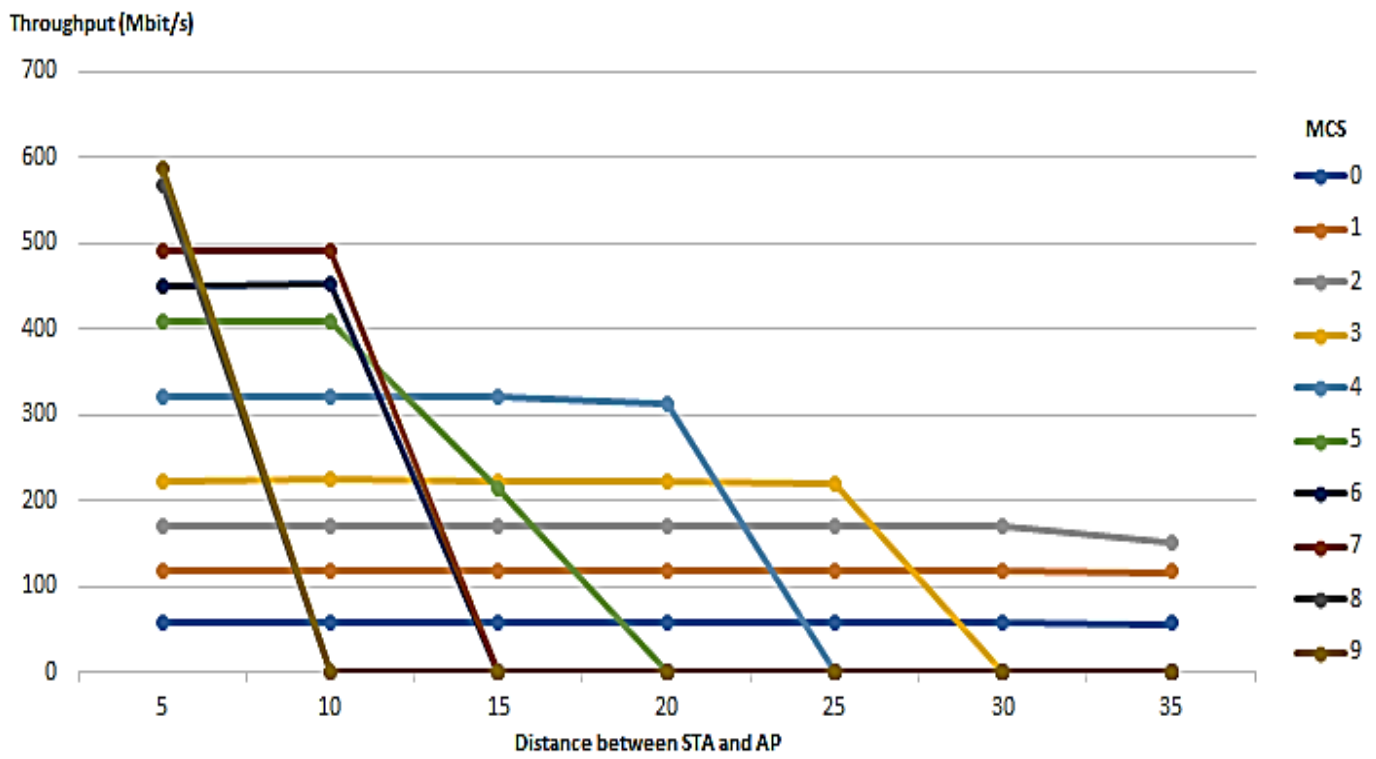

Figure 8. Throughput based on distance (m) 


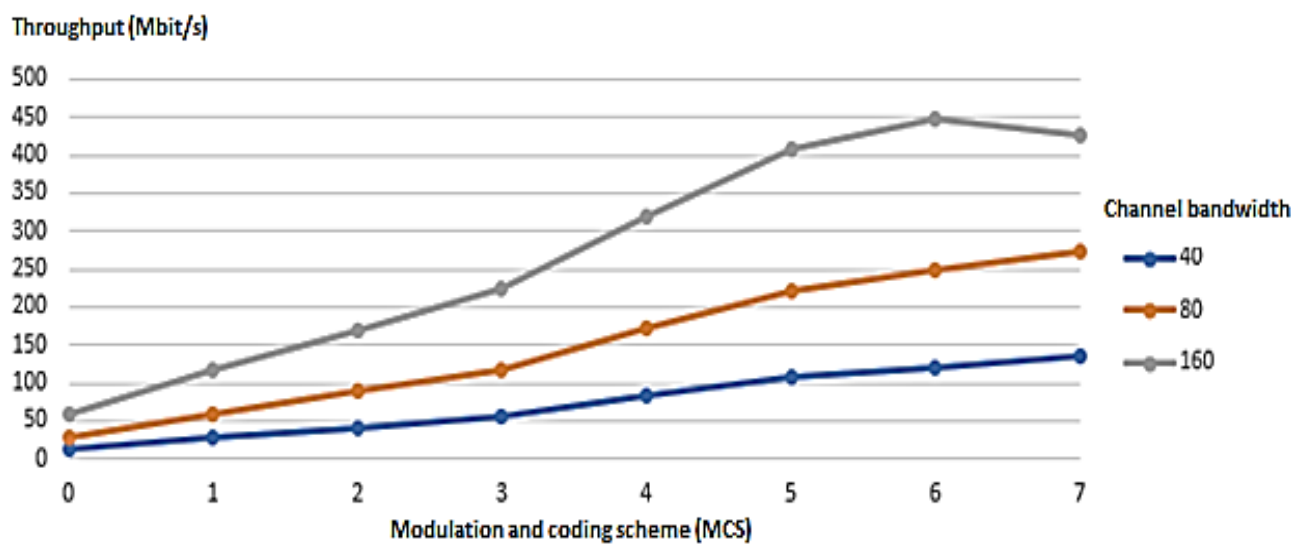

Figure 9. Throughput based on MCS

\section{CONCLUSION}

As seen from the above simulation results, 802.11ac standard can achieve throughputs in the order of $600 \mathrm{Mbit} / \mathrm{s}$ in real scenario approaching the data rates defined in IEEE 802.11ac standard specification. Also, we have shown the effects of shrinking guard interval in 802.11ac on throughput for several channel bandwidths. Then we presented the performance of hybrid frame aggregation that allows the highest throughputs comparing to A-MPDU or A-MSDU aggregations. At last, we analyzed the most suitable modulation and coding schemes according to the distance between STA and the access point and we revealed that for longer ranges, QPSK modulation delivers better performance than 256QAM. Finally, as a future work, the performance of the network will be further examined by considering the quality of service enhancements and comparing the outcomes with the performance of the high efficiency wireless network 802.11ax.

\section{REFERENCES}

[1] G. Bianchi, "Performance Analysis of the IEEE 802.11 Distributed Coordination Function," IEEE Journal on Selected Areas in Communications, vo1. 8, no. 3, pp. 535-547, 2000.

[2] Z. Machrouh and A. Najid, "Performance Analysis of IEEE802.11ac DCF Enhancement for VHT with Frame Aggregation," International Journal of Recent Contributions from Engineering, Science \& IT, vol. 4, no. 3, pp. 17-21, 2016.

[3] Z. Machrouh and A. Najid, "Performance Analysis of IEEE 802.11ac Very High Throughput at MAC and PHY Layers with Frame Aggregation," International Journal on Communications Antenna and Propagation, vol. 7, no. $5,2017$.

[4] A. Z. Yonis, "Performance analysis of IEEE 802.11ac based WLAN in wireless communication systems," International Journal of Electrical and Computer Engineering, vol. 9, no. 2, pp. 1131-1136, 2019.

[5] N. S. Ravindranath, Inder Singh, Ajay Prasad and V. S. Rao, "Performance Evaluation of IEEE 802.11ac and 802.11n using NS3," Indian Journal of Science and Technology, vol. 9, no. 26, pp. 1-8, 2016.

[6] Lee WH and Hwang HY, "A-MPDU aggregation with optimal number of MPDUs for delay requirements in IEEE 802.11ac," PLOS ONE, vol. 14, no. 3, pp. 1-17, 2019.

[7] H. M. H. Herrera, O. J. S. Parra, and J. B. Velandia, "Performance Analysis in WLAN Networks using 802.11ac Technology," International Journal of Applied Engineering Research, vol. 13, no. 7, pp. 5477-5483, 2018.

[8] Matthew S. Gast, "802.11ac: A Survival Guide," O'Reilly Media, Inc., 2013.

[9] White Paper, "802.11ac Demystified," Xirrus Inc., 2013.

[10] Ruizhi Liao, Boris Bellalta, Jaume Barcelo, Victor Valls and Miquel Oliver, "Performance analysis of IEEE 802.11 ac wireless backhaul networks in saturated conditions," EURASIP Journal on Wireless Communications and Networking, vol. 2013, no. 1, pp. 1-14, 2013.

[11] Joseph Kampeas, Asaf Cohen and Omer Gurewitz, "Analysis of Different Approaches to Distributed Multiuser MIMO in the 802.11ac," IEEE Transactions on Mobile Computing, pp. 1-14, 2019.

[12] A. Goldsmith, S. A. Jafar, N. Jindal, and S. Vishwanath, "Capacity limits of MIMO channels," IEEE Journal on Selected Areas in Communications, vol. 21, no. 5, pp. 684-702, 2003.

[13] G. Caire and S. Shamai, "On the achievable throughput of a multiantenna Gaussian broadcast channel," IEEE Transactions on Information Theory, vol. 49, no. 7, pp. 1691-1706, 2003.

[14] D. Gesbert, M. Kountouris, R. W. Heath, C.-B. Chae, and T. Salzer, "Shifting the MIMO paradigm: From single user to Multiuser Communications," IEEE Signal Processing Magazine, vol. 24, no. 5, pp. 36-46, 2007.

[15] Ruizhi Liao, Boris Bellalta, Miquel Oliver, and Zhisheng Niu, "MU-MIMO MAC Protocols for Wireless Local Area Networks: A Survey," IEEE Communications Surveys \& Tutorials, vol. 18, no. 1, pp. 162-183, 2014. 
[16] Yousri Daldoul, Djamal-Eddine Meddour and Adlen Ksentini, "An Analytical Comparison of MU-MIMO and Single User Transmissions in IEEE 802.11ac," IEEE 30th Annual International Symposium on Personal, Indoor and Mobile Radio Communications (PIMRC), pp. 1-6, 2019.

[17] White Paper, "802.11ac In-Depth," Aruba Networks, 2012.

[18] Ziyad Khalaf Farej and Mustafa Mohammad Jasim, "Performance evaluation of the IEEE 802.11n random topology WLAN with QoS application," International Journal of Electrical and Computer Engineering, vol. 10, no. 2, pp. 1924-1934, 2020.

[19] Technical White Paper, "802.11ac: The Fifth Generation of Wi-Fi," Cisco, 2014.

[20] B. T. Vijay and B. Malarkodi, "MAC improvements for very high throughput WLANs," International Journal of Communication Networks and Distributed Systems, vol. 22, no. 1, pp. 74-97, 2019.

[21] M. Inamullah and B. Raman, "802.11ac Frame Aggregation is Bottlenecked: Revisiting the Block ACK," Conference: the 22nd International ACM Conference, pp. 45-49, 2019.

[22] Raja Karmakar, Samiran Chattopadhyay, and Sandip Chakraborty, Members of IEEE, "Impact of IEEE 802.11n/ac PHY/MAC High Throughput Enhancements on Transport and Application Protocols," IEEE communications surveys \& tutorials, vol. 19, no. 4, pp. 2050-2091, 2017.

[23] Subhransu Das, Pushpendu Kar and Subhabrata Barman, "Analysis Of IEEE 802.11 WLAN Frame Aggregation Under Different Network Conditions," IEEE WiSPNET conference, pp. 1240-1245, 2017.

[24] Tutorial, "NS3 simulator," [Online], Available: https://www.nsnam.org.

[25] Rajan Patel, and Pariza Kamboj, "Investigation of NetworkSimulation Tools and Comparison Study: NS3 vs NS2," Journal of Network Communications and Emerging Technologies, vol. 5, no. 2, 2015.

\section{BIOGRAPHIES OF AUTHORS}

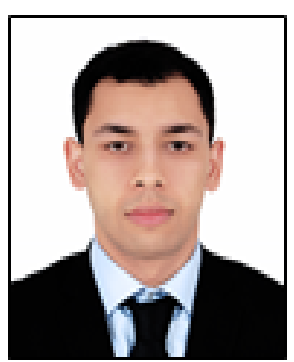

Nabil Khalil was born, on December 1988. He received the Telecommunications and networks engineering degree in 2011 from the National School of Applied Science (ENSA), Sidi Mohamed Ibn Abdullah University, Fez, Morocco. KHALIL Nabil Worked in a telecommunications operator and currently works as a Telecommunications engineer at the National Telecommunications Regulatory Agency (ANRT), Rabat, Morocco. Member of the International Telecommunication Union and $\mathrm{PhD}$ student at National Institute of posts and Telecommunications, Rabat, Morocco.

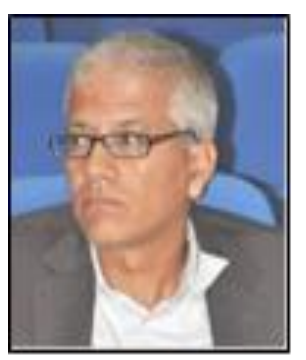

Abdellah Najid received the Ph.D. degree in electronic engineering and the MS degree in Networking and Communication Systems from the ENSEEIHT of Toulouse, France. He has several years of research experience with ENSEEIHT, ENSTA and INRIA. Dr. Abdellah NAJID joined INPT Rabat Morocco as full professor of Microwave and Telecommunication engineering in 2000. He has devoted over 16 years to teaching microwave engineering; Wireless Networking; Network Architectures; Network Modeling courses and directing research projects in Wireless Network performance analysis; Wireless Sensor Networking; Microwave and antennas design. 